\title{
Long non-coding RNA DANCR promotes malignant phenotypes of bladder cancer cells by modulating the miR-149/MSI2 axis as a ceRNA
}

Yonghao Zhan®, Zhicong Chen, Yifan Li, Anbang He, Shiming He, Yanqing Gong, Xuesong Li ${ }^{*}$ and Liqun Zhou*

\begin{abstract}
Background: Accumulating evidences have indicated that long non-coding RNAs (IncRNAs) are potential biomarkers that play key roles in tumor development and progression. Differentiation antagonizing non-protein noding RNA (DANCR) is a novel IncRNA that acts as a potential biomarker and is involved in the development of cancers. However, the clinical significance and molecular mechanism of DANCR in bladder cancer is still unknown.

Methods: The relative expression level of DANCR was determined by Real-Time qPCR in a total of 106 patients with urothelial bladder cancer and in different bladder cancer cell lines. Loss-of-function experiments were performed to investigate the biological roles of DANCR on bladder cancer cell proliferation, migration, invasion and tumorigenicity. Comprehensive transcriptional analysis, RNA-FISH, dual-luciferase reporter assay and western blot were performed to explore the molecular mechanisms underlying the functions of DANCR.
\end{abstract}

Results: In this study, we found that DANCR was significantly up-regulated in bladder cancer. Moreover, increased DANCR expression was positively correlated with higher histological grade and advanced TNM stage. Further experiments demonstrated that knockdown of DANCR inhibited malignant phenotypes and epithelial-mesenchymal transition (EMT) of bladder cancer cells. Mechanistically, we found that DANCR was distributed mostly in the cytoplasm and DANCR functioned as a miRNA sponge to positively regulate the expression of musashi RNA binding protein 2 (MSI2) through sponging miR-149 and subsequently promoted malignant phenotypes of bladder cancer cells, thus playing an oncogenic role in bladder cancer pathogenesis.

Conclusion: This study is the first to demonstrate that DANCR plays a critical regulatory role in bladder cancer cell and DANCR may serve as a potential diagnostic biomarker and therapeutic target of bladder cancer.

Keywords: DANCR, miR-149, ceRNA, MSI2, Bladder cancer

\section{Background}

Urothelial carcinoma of the bladder (BC) is the sixth most common malignancy in men and the most common genitourinary malignancy worldwide; its incidence and mortality have significantly increased over the past decade [1-3]. Although clinical treatments have improved over the past decade, including surgery, radiation therapy, chemotherapy, and immunotherapy, the prognosis of

\footnotetext{
* Correspondence: pineneedle@sina.com; zhoulqmail@sina.com

* Correspondence: pineneedle@sina.com; zhoulqmail@sina.com Urology, Peking University, National Urological Cancer Centre, No. 8 Xishiku street, Beijing 100034, China
}

(c) The Author(s). 2018 Open Access This article is distributed under the terms of the Creative Commons Attribution 4.0 International License (http://creativecommons.org/licenses/by/4.0/), which permits unrestricted use, distribution, and reproduction in any medium, provided you give appropriate credit to the original author(s) and the source, provide a link to the Creative Commons license, and indicate if changes were made. The Creative Commons Public Domain Dedication waiver (http://creativecommons.org/publicdomain/zero/1.0/) applies to the data made available in this article, unless otherwise stated.

patients diagnosed with $\mathrm{BC}$ has not significantly improved [4-6]. The prognosis of bladder cancer is closely related to the stage of disease, but patients do not have specific symptoms at the early stage of bladder cancer $[7,8]$. Therefore, finding promising early detection markers and more efficient and safer therapeutic methods have enormous potential significance for improving the clinical strategies and outcomes of bladder cancer.

The long non-coding RNAs (lncRNAs) are an important groups of transcribed RNA molecules that have a length greater than 200 nucleotides [9-11]. The rapid development of RNA genomics has highlighted the role of 
lncRNAs in many human diseases, especially in cancers [12-15]. Recent accumulating evidences have indicated that lncRNAs, such as SPRY4-IT1, UCA-1, and PANDAR, play important regulatory roles in diverse biological processes in bladder cancer [16-22]. Differentiation antagonizing non-protein noding RNA (DANCR, HGNC:28964) is a novel identified lncRNA located at 4q12.5 [23-26]. Recently, DANCR originally was identified as a potential biomarker and was involved in the development of multiple cancers [27-33]. Although DANCR has been suggested to act as an oncogene, the underlying mechanism by which DANCR-mediated gene expression participates in tumorigenesis remains to be clarified $[34,35]$. However, the biological function and underlying mechanism of DANCR in bladder cancer is completely unknown.

In the present study, we showed that DANCR was significantly up-regulated in bladder cancer tissues compared with corresponding non-tumor tissues in a cohort of 106 bladder cancer patients and its expression was significantly correlated with histological grade and TNM stage. Further experiments demonstrated that knockdown of DANCR inhibited malignant phenotypes (proliferation, migration, invasion and EMT and tumorigenicity) of bladder cancer cells. Mechanistically, we found that DANCR was distributed mostly in the cytoplasm and DANCR functioned as a miRNA sponge to positively regulate musashi RNA binding protein 2 (MSI2) expression by sponging miR-149 in a ceRNA-dependent manner. Moreover, knockdown of miR149 reversed the inhibition of MSI2 expression and MSI2 overexpression reversed the malignant phenotype inhibition of bladder cancer cells induced by silencing DANCR. Together, our results suggest that DANCR is a powerful tumor biomarker, which highlight its potential clinical utility as a promising diagnostic and therapeutic target of bladder cancer.

\section{Methods}

\section{Patients and clinical samples collection}

Between 2011 and 2016, A total of 106 bladder cancer patients who had undergone radical cystectomy without preoperative therapy were enrolled in this study (79 $74.5 \%$ males; median age 63 years) (Additional file 1: Table S1). Fresh bladder cancer tissue samples and pair-matched normal tissue samples were obtained from patients who underwent radical cystectomy. After resection fresh bladder cancer tissue and pair-matched normal adjacent bladder tissue obtained from the same patient were snap-frozen in liquid nitrogen immediately. All patients included in this study signed informed consent and this study was approved by the Institutional Review Board of Peking University First Hospital, Beijing, China.

\section{Bladder cancer cell lines and cell culture}

Bladder cancer 5637, SW780, UM-UC-3, T24 and SVHUC-1 cells used in this study were purchased from the Institute of Cell Research, Chinese Academy of Sciences, Shanghai, China. The UM-UC-3, T24 and SV-HUC-1 cells were cultured in Dulbecco's Modified Eagle Medium (Invitrogen, Carlsbad, CA, USA) plus 10\% fetal bovine serum. The SW780 and 5637 cells were cultured in RPMI-1640 Medium (Invitrogen, Carlsbad, CA, USA) plus $10 \%$ fetal bovine serum. Corresponding plates were placed at $37{ }^{\circ} \mathrm{C}$ with a humidified atmosphere of $5 \% \mathrm{CO} 2$ in incubator.

\section{Cell transfection}

Plasmid vector PLKO.1-puro and pLVX-EF1 $\alpha$ were purchased from BioVector NTCC Inc., Guangzhou, China. The sequences of the related DANCR-shRNA and the negative control were designed and chemically synthesized. These synthetic related sequences were inserted into PLKO.1-puro vector. The sequences of MSI2 and the negative control were chemically synthesized and inserted into pLVX-EF1 $\alpha$ vector. The microRNA mimics (agomir) and microRNA inhibitor (antagomir) were purchased from RIBOBIO, Guangzhou, China. Before transfection, the cells were cultured $24 \mathrm{~h}$. Then, the cells were transiently transfected with corresponding vector using Lipofectamine 3000 Transfection Reagent (Invitrogen, Carlsbad, CA, USA) according to the manufacturer's instructions. After $48 \mathrm{~h}$, cells transfected with corresponding vector were harvested for quantitative real-time PCR. Experiments were repeated at least three times.

\section{RNA extraction and quantitative real-time PCR}

The total RNA of the tissue samples and the transfected cells were extracted using the Trizol reagent (Invitrogen, Carlsbad, CA, USA) according to the manufacturer's instructions. The detailed primer sequences included in this study are shown in Additional file 2: TableS2. Quantitative real-time PCR was performed using the ABI PRISM 7000 Fluorescent Quantitative PCR System (Applied Biosystems, Foster City, CA, USA) according to the manufacturer's instructions and normalized to $\beta$-actin or U6 small nuclear RNA. Experiments were repeated at least three times.

\section{Western blotting analysis}

Total cell lysates were prepared in a $1 \times$ sodium dodecyl sulfate buffer. Total protein was separated by sodium dodecyl sulfate-polyacrylamide gel electrophoresis and transferred onto nitrocellulose membranes. Then the membrane was blocked with $5 \%$ non-fat milk and incubated with primary antibodies at $4{ }^{\circ} \mathrm{C}$ overnight. After incubation with antibodies specific for MSI2, E-cadherin, N-cadherin, vimentin (Abcam, Hong Kong, China), the blots were incubated with goat anti-rabbit secondary antibody (Abcam, 
Hong Kong, China) and visualized with enhanced chemiluminescence. Experiments were repeated at least three times.

\section{Cell counting Kit-8 assay}

Cell proliferation was determined using Cell Counting Kit-8 (Beyotime Inst Biotech, China) according to the manufacturer's instructions. Briefly, $5 \times 103$ cells/well were seeded in a 96-well flat-bottomed plate, and grown at $37^{\circ} \mathrm{C}$ for $24 \mathrm{~h}$, then transfected with corresponding vector. Finally, the absorbance was finally determined at a wavelength of $450 \mathrm{~nm}$ using a microplate reader (Bio-Rad, Hercules, CA, USA). Experiments were repeated at least three times.

\section{Ethynyl-2-deoxyuridine (EdU) incorporation assay}

Cell proliferation was also determined by Ethynyl-2deoxyuridine incorporation assay using an EdU Apollo DNA in vitro kit (RIBOBIO, Guangzhou, China) following the manufacturer's instructions. Briefly, after transfected with corresponding vector cells were incubated with $100 \mu \mathrm{l}$ of $50 \mu \mathrm{M}$ EdU per well for $2 \mathrm{~h}$ at $37^{\circ} \mathrm{C}$, respectively. Finally, the cells were visualized under a fluorescence microscopy. Experiments were repeated at least three times.

\section{Wound healing assay}

Cell motility was determined by wound healing assay. At $24 \mathrm{~h}$ post transfection, a wound field was created using a sterile $200 \mu \mathrm{l}$ pipette tip in about $90 \%$ confluent cells. The cells were incubated for $24 \mathrm{~h}$ at $37^{\circ} \mathrm{C}$, and then the migration of cells was monitored with a digital camera system. The cell migration distance $(\mu \mathrm{m})$ was calculated by the software program HMIAS-2000. Experiments were repeated at least three times.

\section{Transwell assay}

The invasion of bladder cancer cells was determined using a transwell insert $(8 \mu \mathrm{m}$, Corning). $24 \mathrm{~h}$ after transfection, $5 \times 10^{4}$ cells were first starved in $200 \mathrm{ml}$ serum free medium and then placed in the dishes. The lower chamber was filled with $500 \mathrm{ml}$ of complete medium. The cells were incubated for $48 \mathrm{~h}$ at $37{ }^{\circ} \mathrm{C}$, and then the cells that had migrated to the bottom surface of the filter membrane were stained with $0.5 \%$ crystal violet solution and photographed. Finally, the absorbance was determined at a wavelength of $570 \mathrm{~nm}$ using a microplate reader (Bio-Rad, Hercules, CA, USA). Experiments were repeated at least three times.

\section{Immunofluorescence}

Immunostaining was performed on the paraffin-embedded tumor tissues from nude mice. The avidin-biotin-peroxidase method was adopted to determine the location and relative expression level of the target proteins. The primary antibodies of MSI2, E-cadherin, N-cadherin, vimentin were used at a dilution of 1:2000. Sections were visualized under a fluorescence microscopy (Olympus, Japan). Experiments were repeated at least three times.

\section{RNA fluorescent in situ hybridization (FISH)}

FISH assay was performed using Ribo ${ }^{\text {tw }}$ Fluorescent in Situ Hybridization Kit (Ribobio Company, China). DANCR and U6 probes were designed and synthesized by Ribobio Company and labeled with Cy3 fluorescent dye. RNA FISH were performed using fluorescent in situ hybridization kit (RiboBio) following the manufacturer's instructions. Fluorescence detection was performed with a confocal laser-scanning microscope (Leica, Germany).

\section{Dual-luciferase reporter assay}

MSI2-WT/MUT (GenePharma, Shanghai, China) were constructed and transfected into T24 along with Agomir-149b/ Agomir-NC. Luciferase activity was detected using the Dual-Luciferase Reporter Assay System (Promega; $48 \mathrm{~h}$ after transfection) according to the manufacturer's instructions. Experiments were repeated at least three times.

\section{Tumor xenograft implantation in nude mice}

Animal work was permitted by the Institutional Animal Care and Use Committee (IACUC) of Peking University First Hospital (Beijing, China), and conducted in accordance with its recommendations and ethical regulations. The mice were maintained under standard conditions according to the institutional guidelines for animal care. BCCs were collected after transfection for 48 h. $5 \times 10^{5}$ BCCs were injected subcutaneously into BALB/c-Nude mice. The mice were euthanized after 8 weeks.

\section{Statistical analyses}

All experimental data from three independent experiments were analyzed by Student's t-test or $\mathrm{X} 2$ test and results were expressed as mean \pm standard deviation. P-values of less than 0.05 were considered to be statistically significant. All statistical tests were conducted by SPSS version 19.0 software (SPSS Inc. Chicago, IL, USA).

\section{Results \\ DANCR expression is up-regulated in bladder cancer tissues and cell lines}

The relative expression level of DANCR was determined by qRT-PCR in bladder cancer tissues and cell lines. The CASC9 expression fold change (bladder cancer tissue/ matched normal tissue) in each patient was indicated in Fig. 1a. DANCR expression was up-regulated in $66.05 \%$ (70/106) of bladder cancer tissues compared with the corresponding normal tissue samples (Fig. 1b, c). Moreover, elevated DANCR expression was positively correlated with advanced TNM stage (Fig. 1d) and higher histological grade 

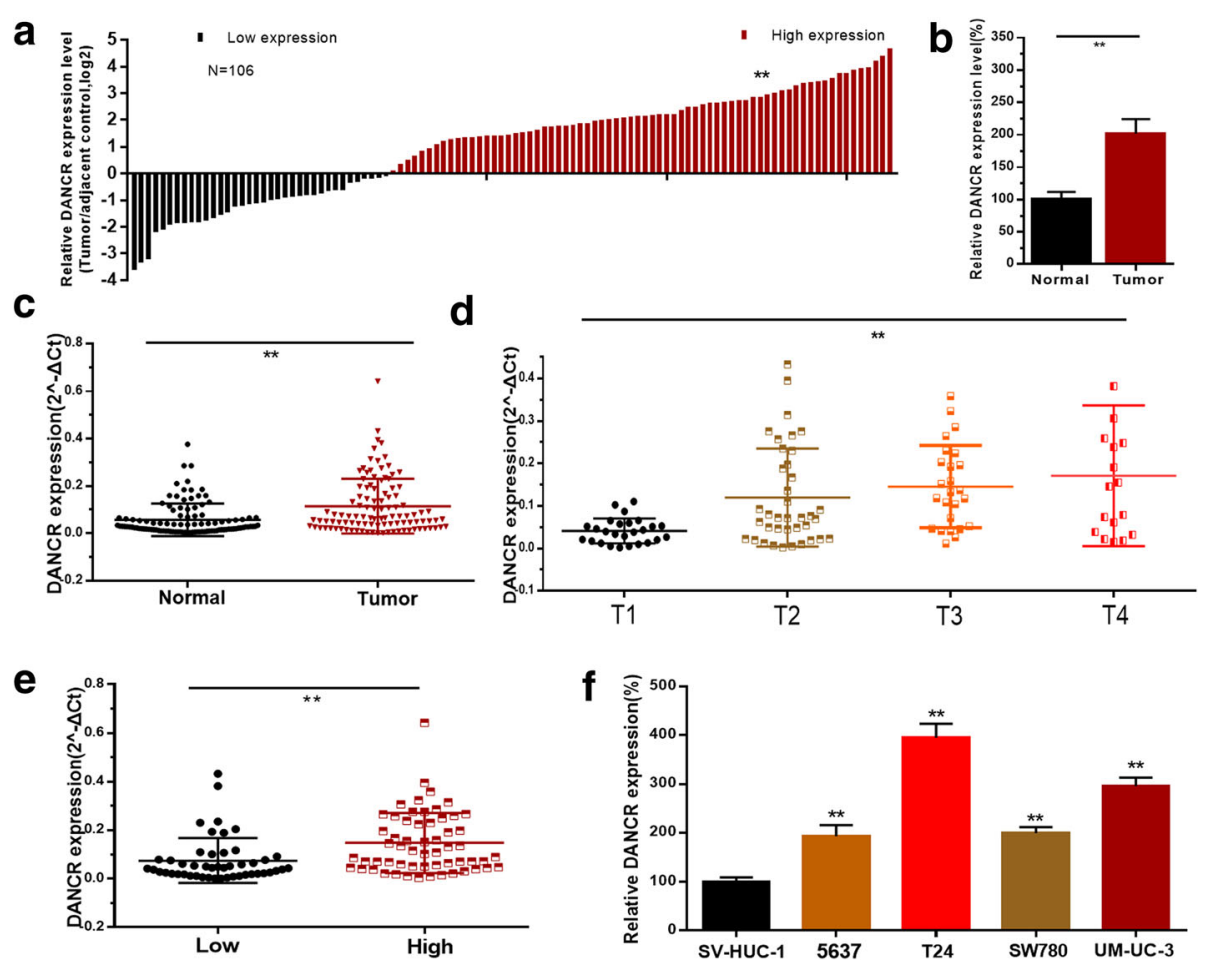

Fig. 1 The relative expression levels of DANCR in bladder cancer. a: The heights of the columns in the chart represent the log2-transformed fold changes (bladder cancer tissue/normal bladder tissue) in DANCR expression in 106 patients with bladder cancer. $\mathbf{b}$ and $\mathbf{c}$ : DANCR is up-regulated in bladder cancer tissues compared with corresponding non-tumor tissues. $\mathbf{d}$ : DANCR is up-regulated in bladder cancer patients with advanced TNM stage. e: DANCR is up-regulated in bladder cancer patients with higher histological grade. f: DANCR is up-regulated in bladder cancer cell lines compared to normal urothelial cell line. Data are shown as mean \pm SD. ${ }^{*} p<0.05 ;{ }^{* *} p<0.01$

(Fig. 1e). DANCR expression was up-regulated in BC cell lines compared with the normal urothelial cell line SV-HUC-1 (Fig. 1f). Clinicopathological features of patients and statistical results are shown in Table 1 and Additional file 1: Table S1, respectively.

\section{Knockdown of DANCR inhibits cell proliferation of bladder cancer cells}

We further determined whether DANCR regulated cell proliferation of bladder cancer cells. The DANCR specific shRNAs significantly down-regulated the expression level of DANCR in T24 and UM-UC-3 cells (Fig. 2a). The cell proliferation changes of bladder cancer cells were determined using CCK-8 assay, colony-formation assays and Edu assay. Inhibited cell proliferations were both observed in T24 and UM-UC-3 cells by silencing DANCR (Fig. 2b-f). These results demonstrated that DANCR promotes cell proliferation of bladder cancer cells.

\section{Knockdown of DANCR inhibits cell migration, invasion and EMT of bladder cancer cells}

We further determined whether DANCR regulated cell migration and invasion of bladder cancer cells. The migratory abilities of bladder cancer cells were determined using wound healing assay. Inhibited cell migrations were observed in T24 and UM-UC-3 induced by silencing DANCR (Fig. 3a, b). The invasive abilities of bladder cancer cells were determined using transwell assay. Inhibited cell invasions were observed in T24 and UM-UC-3 induced by silencing DANCR (Fig. 3c, d). We further determined whether DANCR regulated EMT of bladder cancer cells. The expression of EMT markers were determined using qRT-PCR, western blotting and immunofluorescence. Knockdown of DANCR increased E-cadherin expression and decreased N-cadherin and vimentin expression in bladder cancer cells (Fig. 3e, f, g). The results indicated that DANCR promotes cell migration, invasion and EMT of bladder cancer cells.

\section{DANCR positively regulates MSI2 expression via sponging miR-149}

The subcellular localization of lncRNAs is closely related to their biological function and potential molecular roles. First, we detected the subcellular localization of DANCR using RNA-FISH. The results showed that most of the positives orientated in the cytoplasm, minority in the nucleus (Fig. 4a). To investigate the underlying mechanisms of DANCR-mediated biological processes, we performed qRT-PCR and comprehensive transcriptional analysis by using TCGA dataset, CCLE dataset and our dataset. The 
Table 1 Correlation between DANCR expression and clinicopathological features of UCB patients

\begin{tabular}{|c|c|c|c|c|c|}
\hline \multirow[t]{2}{*}{ Parameters Total } & \multirow[t]{2}{*}{ Group } & \multirow[t]{2}{*}{ Total } & \multicolumn{2}{|c|}{ DANCR expression } & \multirow[t]{2}{*}{$P$ value } \\
\hline & & & High & Low & \\
\hline \multirow[t]{2}{*}{ Gender } & Male & $79(75 \%)$ & $55(52 \%)$ & $24(23 \%)$ & \multirow[t]{2}{*}{0.183} \\
\hline & Female & $27(25 \%)$ & $15(14 \%)$ & $12(11 \%)$ & \\
\hline \multirow[t]{2}{*}{ Age (years) } & $<60$ & $37(35 \%)$ & $25(24 \%)$ & $12(11 \%)$ & \multirow[t]{2}{*}{0.808} \\
\hline & $\geq 60$ & $69(65 \%)$ & $45(42 \%)$ & $24(23 \%)$ & \\
\hline \multirow[t]{2}{*}{ Tumor size $(\mathrm{cm})$} & $<3 \mathrm{~cm}$ & $42(40 \%)$ & $26(25 \%)$ & $16(15 \%)$ & \multirow[t]{2}{*}{0.467} \\
\hline & $\geq 3 \mathrm{~cm}$ & $64(60 \%)$ & $44(42 \%)$ & $20(18 \%)$ & \\
\hline \multirow[t]{2}{*}{ Multiplicity } & Single & $59(56 \%)$ & $37(35 \%)$ & $22(21 \%)$ & \multirow[t]{2}{*}{0.418} \\
\hline & Multiple & 47 (44\%) & $33(31 \%)$ & $14(13 \%)$ & \\
\hline \multirow[t]{2}{*}{ Histological grade } & $L$ & $48(46 \%)$ & $25(24 \%)$ & $23(22 \%)$ & \multirow[t]{2}{*}{$0.006^{*}$} \\
\hline & $\mathrm{H}$ & $58(54 \%)$ & $45(42 \%)$ & $13(12 \%)$ & \\
\hline \multirow[t]{2}{*}{ Tumor stage $T$} & $\mathrm{Ta}, \mathrm{T} 1$ & $26(24 \%)$ & $11(10 \%)$ & $15(14 \%)$ & \multirow[t]{2}{*}{$0.003^{*}$} \\
\hline & $\mathrm{T} 2-\mathrm{T} 4$ & $80(76 \%)$ & $59(56 \%)$ & $21(20 \%)$ & \\
\hline \multirow[t]{2}{*}{ Lymph nodes metastasis } & NO & $92(87 \%)$ & $59(56 \%)$ & $33(31 \%)$ & \multirow[t]{2}{*}{0.447} \\
\hline & YES & $14(13 \%)$ & $11(10 \%)$ & $3(3 \%)$ & \\
\hline
\end{tabular}

${ }^{*} P<0.05$ was considered significant (Chi-square test between 2 groups)

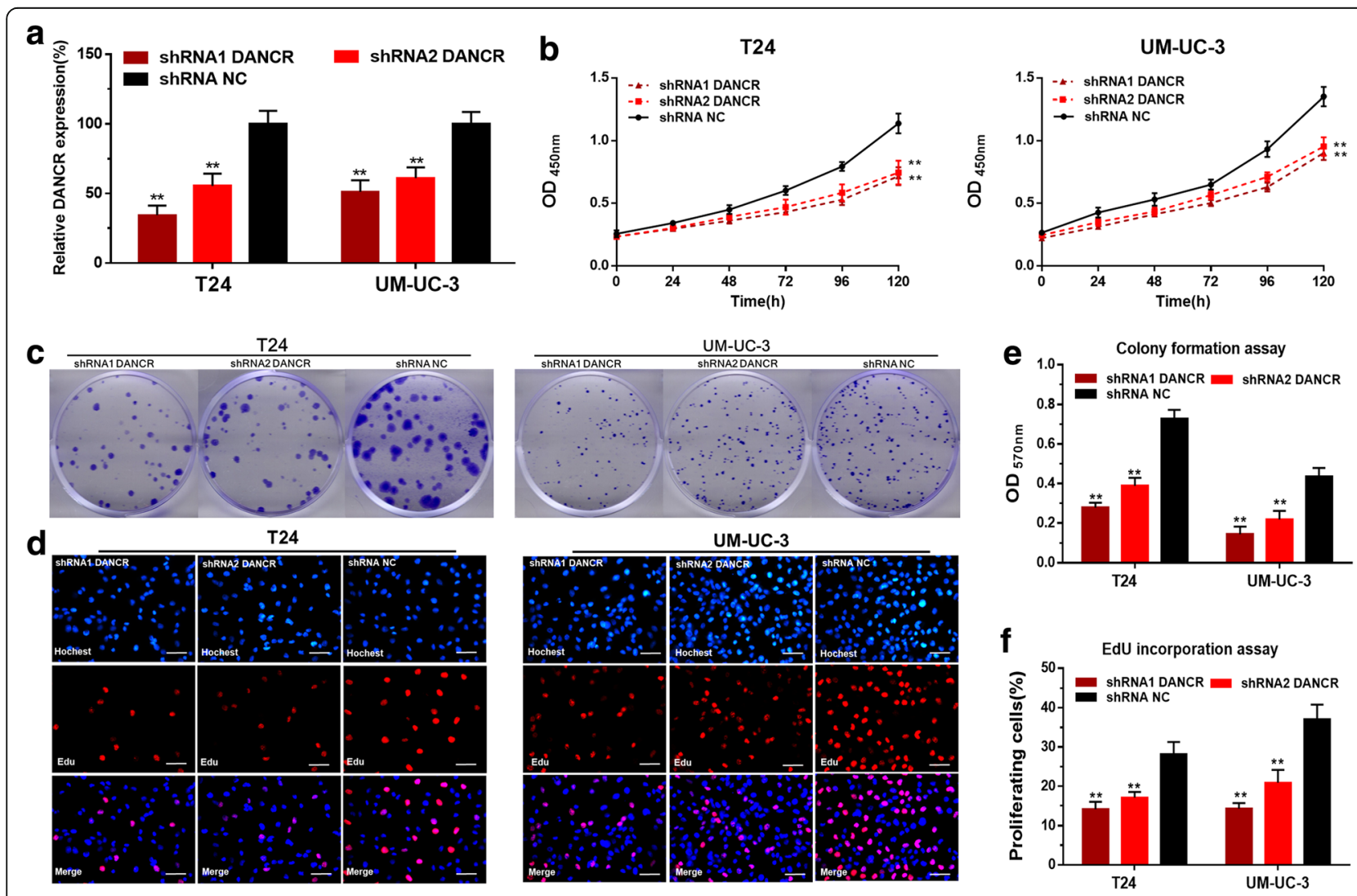

Fig. 2 The effect of DANCR on cell proliferation of bladder cancer cells. a: The DANCR specific shRNAs significantly decreased the expression level of DANCR in T24 and UM-UC-3. b: The cell proliferation changes of bladder cancer cells were determined using CCK-8 assay. c and e: The cell proliferation changes of bladder cancer cells were determined using colony-formation assay. Inhibited cell proliferation by silencing DANCR was observed in T24 and UM-UC-3. d and $\mathbf{f}$ : The cell proliferation changes of bladder cancer cells were determined using Edu assay. Inhibited cell proliferation by silencing DANCR was observed in T24 and UM-UC-3. Data are shown as mean \pm SD. ${ }^{*} p<0.05 ;{ }^{* *} p<0.01$ 


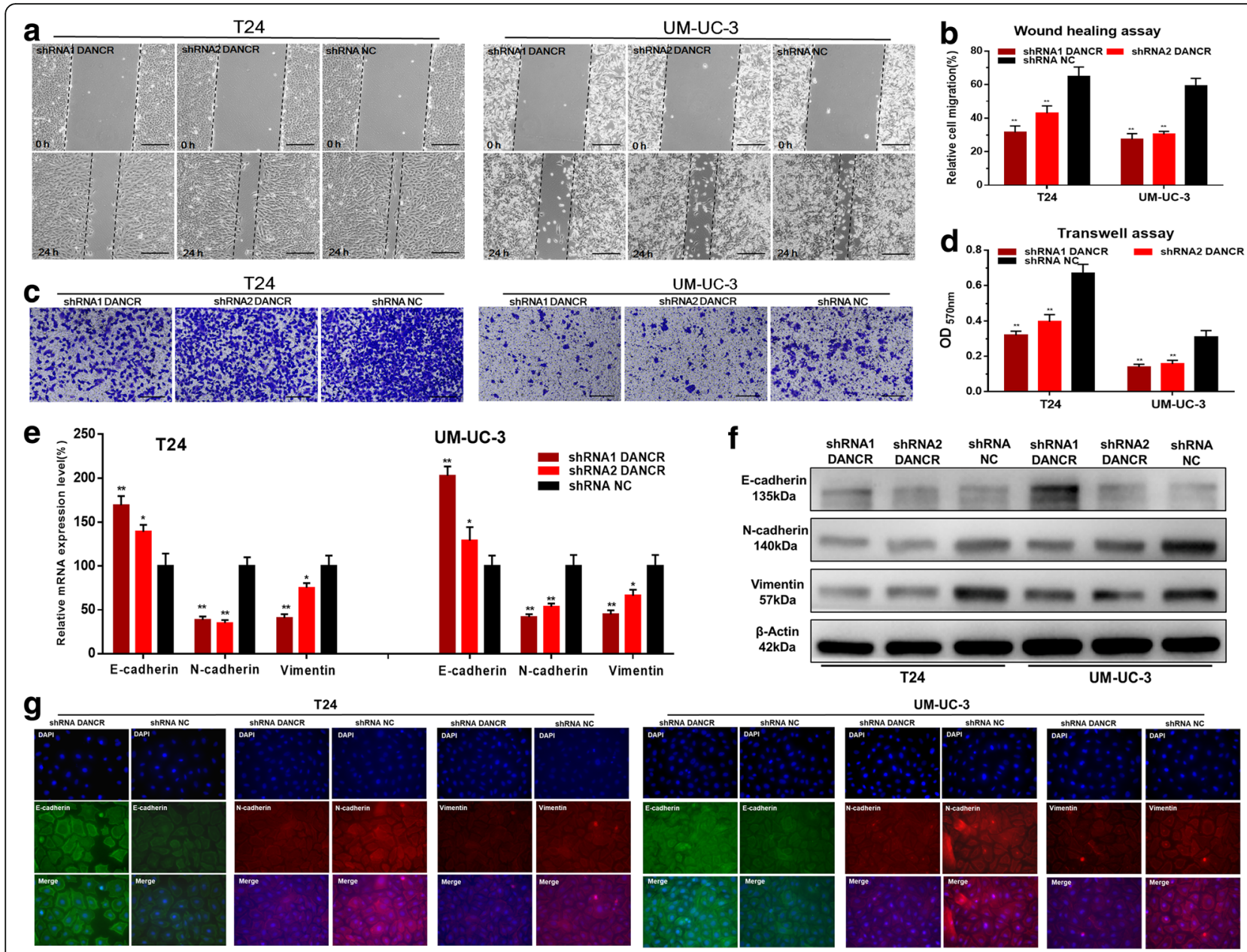

Fig. 3 The effect of DANCR on migration, invasion and EMT of bladder cancer cells. $\mathbf{a}$ and $\mathbf{b}$ : The migratory abilities of bladder cancer cells were determined using wound healing assay. Inhibited cell migration by silencing DANCR was observed in T24 and UM-UC-3. c and d: The invasive abilities of bladder cancer cells were determined using transwell assay. Inhibited cell invasion by silencing DANCR was observed in T24 and UM-UC-3. e-g: The expression of EMT markers were determined using qRT-PCR, western blotting and immunofluorescence. Knockdown of DANCR increased the expression of E-cadherin and decreased the expression of $\mathrm{N}$-cadherin and vimentin in bladder cancer cells. Data are shown as mean $\pm \mathrm{SD}$. ${ }^{*} p<0.05 ;{ }^{* *} p<0.01$

results showed that DANCR expression levels were statistically positively correlated with MSI2 expression levels in bladder cancer (Fig. 4b) and knockdown of DANCR decreased MSI2 expression in bladder cancer cells (Fig. 4c). Through searching online bioinformatics database, bio-information analysis predicted that DANCR and MSI2 had common putative binding sites with multiple miRNAs (Fig. 4d). Detailed prediction results were shown in Additional file 3: Table S3. Then we found knockdown of DANCR increased miR-149 expression in bladder cancer cells (Fig. 4e). Bioinformatics analysis showed that the 3'UTR sequence of MSI2 was complementary to the seed sequence of miR-149 (Fig. 4f). Dual-luciferase reporter assay showed MSI2-Wt and Agomir149 co-transfection significantly inhibited luciferase activity, and MSI2-Mut and Agomir149 co-transfection failed to change luciferase activity (Fig. 4f). Furthermore, knockdown of DANCR decreased the luciferase activity of cells transfected with MSI2-Wt (Fig. 4g). We further determined whether DANCR regulated the expression of MSI2 in BC cells via miR-149-dependent manner. We found overexpressing miR-149 decreased MSI2 expression in BC cells (Fig. 4h). Moreover, knockdown of miR-149 reversed the MSI2 expression inhibition of $\mathrm{BC}$ cells induced by silencing DANCR (Fig. 4i). The results indicated that DANCR positively regulates MSI2 expression via sponging miR-149 in $\mathrm{BC}$ cells.

Overexpressing of MSI2 reverses malignant phenotypes inhibition of bladder cancer cells induced by silencing DANCR

We further determined whether DANCR regulated malignant phenotypes of bladder cancer cells via MSI2dependent manner. Our results showed that the MSI2 


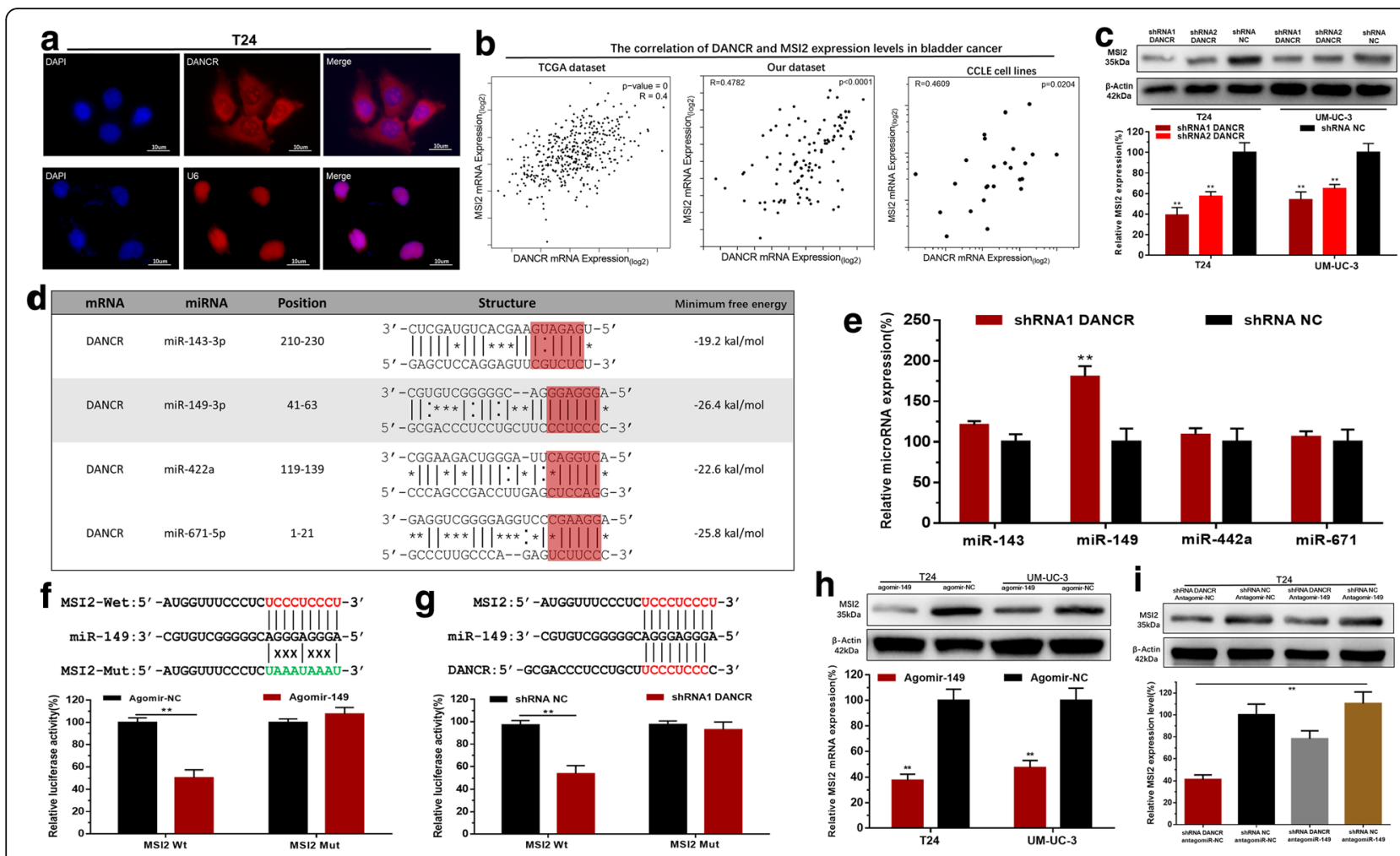

Fig. 4 DANCR positively regulates MSI2 expression via sponging miR-149. a: The RNA-FISH results revealed that DANCR was distributed mostly in the cytoplasm in bladder cancer cells. $\mathbf{b}$ : The comprehensive transcriptional analysis results showed that DANCR expression levels were statistically positively correlated with MSI2 expression levels in bladder cancer. c: Knockdown of DANCR decreased MSI2 expression in bladder cancer cells. d: The bio-information analysis results showed that DANCR and MSI2 have common putative binding sites with multiple miRNAs. e: Knockdown of DANCR increased miR-149 expression in bladder cancer cells. f: Bioinformatics analysis showed that the $3^{\prime} U T R$ sequence of MSI2 is complementary to the seed sequence of miR-149. Dual-luciferase reporter assay showed MSI2-Wt and Agomir149 co-transfection significantly inhibited luciferase activity. g: Knockdown of DANCR decreased the luciferase activity of bladder cancer cells transfected with MSI2-Wt. $\mathbf{h}$ : Overexpressing miR-149 decreased the expression of MSI2 in bladder cancer cells. i: Knockdown of miR-149 increased MSI2 expression in bladder cancer cells transfected with shRNA-DANCR. Data are shown as mean \pm SD. ${ }^{*} p<0.05 ;{ }^{* *} p<0.01$

specific vector (Plvx-MSI2) significantly reversed the inhibition of MSI2 expression induced by silencing DANCR in BC cells (Fig. 5a). Meanwhile, we found MSI2 overexpression significantly reversed cell proliferation inhibition of BC cells (Fig. 5b, c, d) induced by silencing DANCR. Moreover, MSI2 overexpression significantly reversed cell migration (Fig. 5e, f) and invasion (Fig. 5g, h) inhibition of bladder cancer cells induced by silencing DANCR. The results indicated that DANCR promotes malignant phenotypes of bladder cancer cells via MSI2-dependent manner.

\section{Knockdown of DANCR inhibits tumorigenicity of bladder cancer cells}

We further determined whether DANCR regulated tumorigenicity of $\mathrm{BC}$ cells using generation of xenograft. We found knockdown of DANCR inhibited the tumorigenicity of bladder cancer cells in vivo (Fig. 6a-d). Tumors collected from mice were exhibited and measured (Fig. 6a). Tumor growth of NC treatment group was faster than that in the
shDANCR group (Fig. 6b). Tumor weight of NC treatment group was greater than that in the shDANCR group (Fig. 6c). Tumor free mouse proportion of shDANCR group was higher than that in the NC treatment group (Fig. 6d). We found knockdown of DANCR decreased MSI2 expression and inhibited EMT of bladder cancer cells in vivo (Fig. 6e, f). Meanwhile, we found knockdown of DANCR inhibited MSI2 and ki67 expression (Fig. 6g) of bladder cancer cells in vivo. Moreover, we found that DANCR and MSI2 were co-localized in bladder cancer cells (Fig. 6h). The results indicated that DANCR promoted tumorigenicity of bladder cancer cells via upregulating MSI2. As shown in Fig. 7, we found that DANCR was significantly up-regulated in bladder cancer cells and DANCR functioned as a miRNA sponge to positively regulate MSI2 expression through sponging miR-149. Elevated MSI2 protein promoted transcription and translation of proteins operating in essential oncogenic signaling pathways, subsequently promoting malignant phenotypes of bladder cancer cells. 

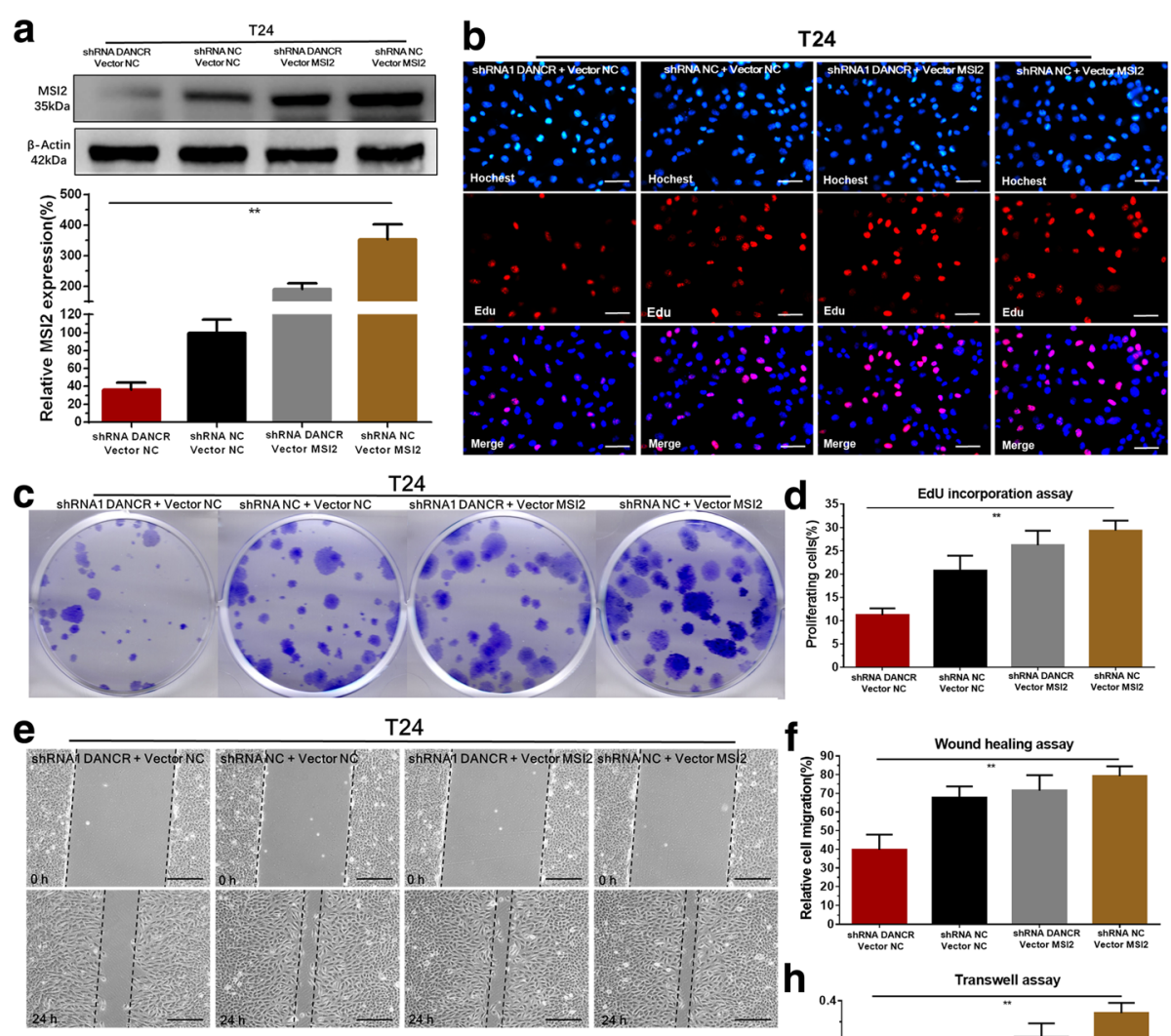

$\mathrm{T} 24$

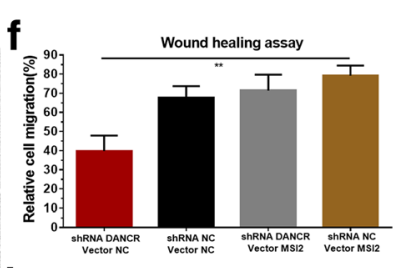

g

T24
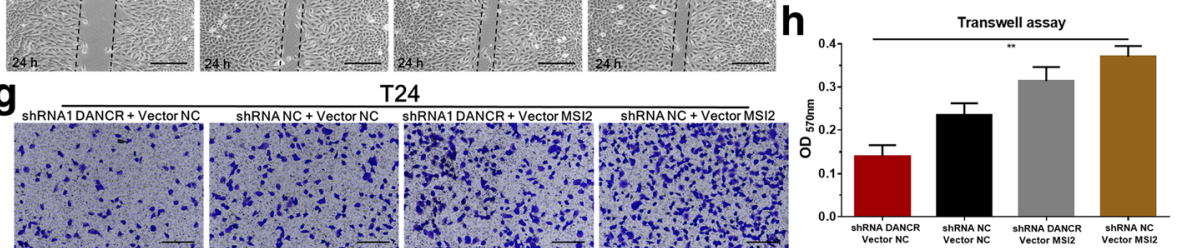

Fig. 5 Overexpressing of MSI2 reversed malignant phenotypes inhibition of bladder cancer cells induced by silencing DANCR. a: The MSI2 specific vector significantly reversed MSI2 expression inhibition induced by silencing DANCR in bladder cancer cells. b-d: Overexpressing MSI2 significantly reversed cell proliferation inhibition induced by silencing DANCR. e and f: Overexpressing MSI2 significantly reversed cell migration inhibition induced by silencing DANCR. $\mathbf{g}$ and $\mathbf{h}$ : Overexpressing MSI2 significantly reversed cell invasion inhibition induced by silencing DANCR. Data are shown as mean \pm SD. ${ }^{*} p<0.05 ;{ }^{* *} p<0.01$

\section{Discussion}

Bladder cancer is the most common genitourinary malignancies all over the world [36, 37]. There are no specific symptoms for patients with bladder cancer at the early stage, therefore, some bladder cancers are diagnosed when treatments are less effective [38-40]. Therefore, finding new prognostic and therapeutic target have enormous potential significance to improving the clinical strategies and outcomes of bladder cancer.

The IncRNAs are important new members of non-coding RNA family, which are longer than 200 nucleotides [41, 42]. Recently, an increasing number of evidences have indicated that lncRNAs regulate gene expression at different processing levels, including chromatin modification, transcription and posttranscriptional regulation [43, 44]. For example, HOTAIR and Xist have also been reported to interact with the chromatin remodeling protein PRC2 complex to repress gene regulation [45, 46].
MALAT1 and ATB have also been reported to downregulate the expression of miR-200c and subsequently increase the expression of ZEB2/ZNF217 in a ceRNA-dependent manner [47, 48]. DANCR is a newly identified lncRNA mapped to human chromosome 4q12.5 locus [49]. Recent studies have provided evidence that DANCR plays a key role in transcription regulation of targeted gene and the disregulation of DANCR expression have become highlighted in some somatic cancers [50-55]. For example, DANCR can interact with the binding site of miR-199a/320a/214 in 3'UTR of CTNNB1 to inhibit the suppression of CTNNB1 by miRNAs, thus promoting stemness features of hepatocellular carcinoma [29]. DANCR competitively binds to miR-335-5p and miR-1972 to regulate the expression of ROCK1, thus promoting the malignant biological behaviours of osteosarcoma [56]. However, the clinical significance and biological function of DANCR in bladder cancer are completely unknown. 

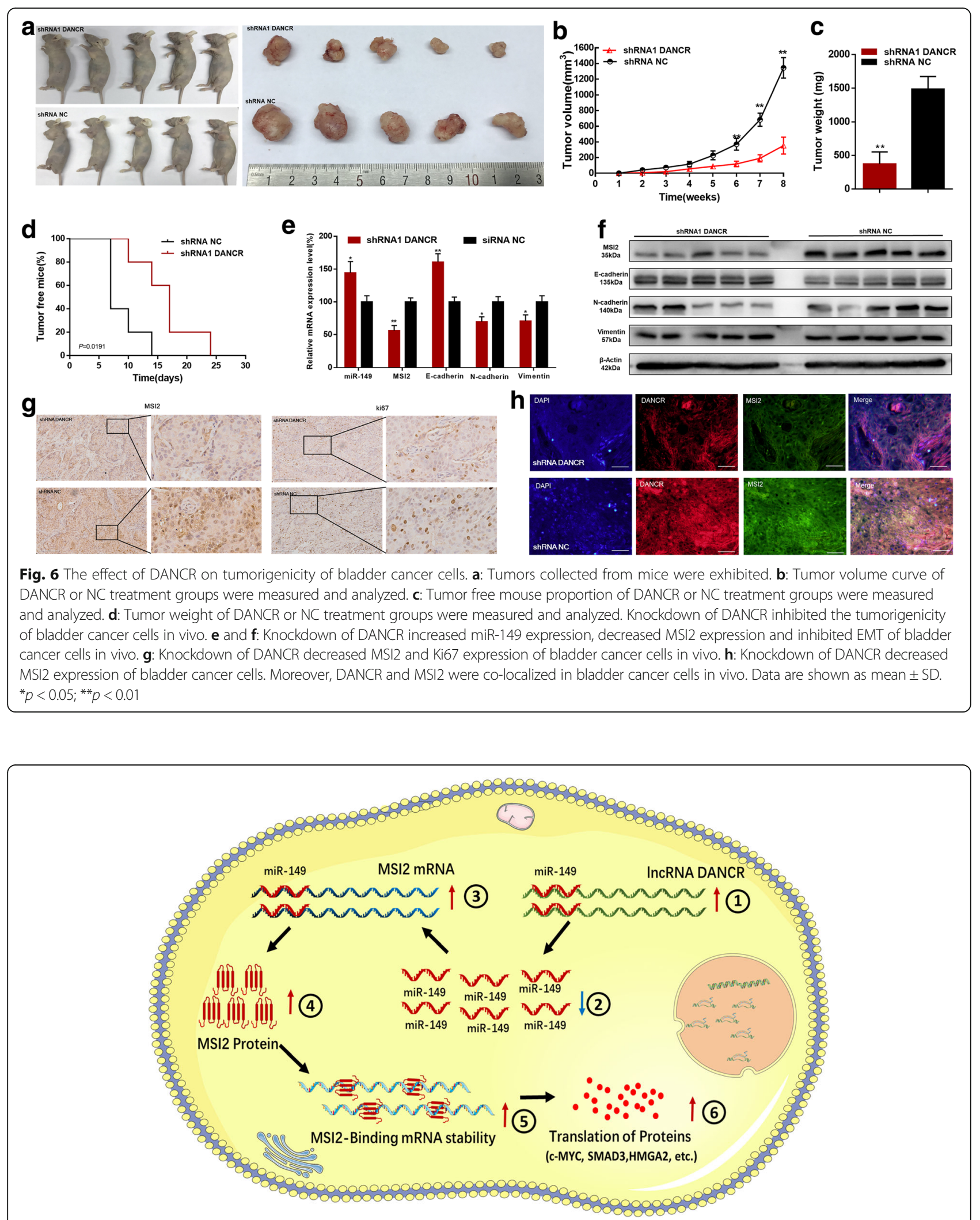

Fig. 7 The schematic diagram of the oncogenic role of DANCR in bladder cancer cells. DANCR functions as a miRNA sponge to positively regulate MSI2 expression through sponging miR-149 and subsequently promotes malignant phenotypes of bladder cancer cells, thus playing an oncogenic role in bladder cancer pathogenesis 
To the best of our knowledge, this is the first report of DANCR being involved in the development of bladder cancer. In this study, we found the expression level of DANCR was significantly up-regulated in bladder cancer tissues compared with corresponding non-tumor tissues and increased DANCR expression was positively correlated with higher histological grade and advanced TNM stage. Meanwhile, the expression level of DANCR was significantly up-regulated in bladder cancer cell lines compared with normal urothelial cell line. Further experiments demonstrated that knockdown of DANCR inhibited malignant phenotypes (proliferation, migration, invasion, EMT and tumorigenicity) of bladder cancer cells. Mechanistically, we found that DANCR expression was statistically positively correlated with MSI2 expression in bladder cancer and knockdown of DANCR decreased MSI2 expression in bladder cancer cells. MSI2 can bind and regulate the mRNA stability and translation of proteins operating in essential oncogenic signaling pathways, such as NUMB/ Notch, PTEN/mTOR, TGF- $\beta /$ SMAD3, MYC, cMET [57]. Based on these biological functions, MSI2 protein can regulate cancer invasion, metastasis and development of more aggressive cancer phenotypes, including drug and radiation resistance [58]. LncRNA subcellular localization patterns can provide fundamental insights into their biology and fosters hypotheses for potential molecular roles. Then we detected the subcellular location of DANCR using RNA-FISH. RNA-FISH results revealed that DANCR was distributed mostly in the cytoplasm, suggesting that DANCR might also play a role in posttranscriptional level in bladder cancer. Through searching online bioinformatics database, bio-information analysis predicted that DANCR and MSI2 have common putative binding sites with miR-149. Further experiments demonstrated that knockdown of DANCR increased miR-149 expression and subsequently inhibited the expression of MSI2 in a ceRNA-dependent manner. Moreover, knockdown of miR-149 reversed MSI2 expression and MSI2 overexpression reversed the malignant phenotype inhibition of bladder cancer cells induced by silencing DANCR.

\section{Conclusions}

Our study reveals that DANCR functions as a miRNA sponge to positively regulate MSI2 expression through sponging miR-149 and subsequently promotes the malignant phenotypes of bladder cancer cells, thus playing an oncogenic role in bladder cancer pathogenesis. The results of this study provide a new basis for studying the mechanism of the occurrence and development of bladder cancer. Cumulatively, our results suggest that DANCR is a powerful tumor biomarker, which highlight its potential clinical utility as a promising diagnostic and therapeutic target of bladder cancer.

\section{Additional files}

Additional file 1: Table S1. Summary of clinicopathological features of tissues of bladder cancer. (DOCX $19 \mathrm{~kb}$ )

Additional file 2: Table2. The primer sequences included in this study. (DOCX $16 \mathrm{~kb}$ )

Additional file 3: Table S3. Results of bioinformation analysis. (DOCX $17 \mathrm{~kb}$ )

\section{Abbreviations}

BC: Bladder cancer; CCLE: Cancer cell line encyclopedia; ceRNA: Competing endogenous RNA; DANCR: Differentiation antagonizing non-protein noding RNA; EdU: Ethynyl-2-deoxyuridine; EMT: Epithelial-mesenchymal transition; FISH: Fluorescent in situ hybridization; InCRNA: Long non-coding RNA; MSI2: Musashi RNA Binding Protein 2; qRT-PCR: Quantitative real time-PCR; TCGA: The Cancer Genome Atlas

\section{Funding}

This work was supported by National Natural Science Foundation of China [81672546, 81602253, 81772703], Natural Science Foundation of Beijing [7152146, 7172219] and The Capital Health Research and Development of Special [2016-1-4077].

Availability of data and materials

The dataset(s) supporting the findings of this study are included within the article.

\section{Authors' contributions}

$\mathrm{ZL}$ and $\mathrm{LX}$ contributed to the experiment design, manuscript draft, and data analysis. ZY and LY contributed to the experiment implementation, manuscript draft and data analysis. HS and GY contributed to the experiment implementation and data analysis. CZ and HA collected clinical samples. All authors read and approved the final manuscript.

\section{Ethics approval and consent to participate}

This study was approved by the ethics committee of the Peking University First Hospital and written informed consents were obtained before any operation to patients. Consent for publication The authors confirmed that we have obtained written consent from the patients to publish this manuscript.

\section{Consent for publication}

The authors confirmed that we have obtained written consent from the patients to publish this manuscript.

\section{Competing interests}

We declare that we have no financial and personal relationships with other people or organizations that can inappropriately influence our work.

\section{Publisher's Note}

Springer Nature remains neutral with regard to jurisdictional claims in published maps and institutional affiliations.

Received: 20 July 2018 Accepted: 28 September 2018 Published online: 12 November 2018

\section{References}

1. Dy GW, Gore JL, Forouzanfar MH, Naghavi M, Fitzmaurice C. Global burden of urologic cancers, 1990-2013. Eur Urol. 2017;71:437-46.

2. Grayson M. Bladder cancer. Nature. 2017;551:S33.

3. Berdik C. Bladder cancer: 4 big questions. Nature. 2017;551:S51.

4. Humphrey PA, Moch H, Cubilla AL, Ulbright TM, Reuter VE. The 2016 WHO classification of Tumours of the urinary system and male genital organs-part B: prostate and bladder Tumours. Eur Urol. 2016;70:106-19.

5. Alfred Witjes J, Lebret T, Comperat EM, Cowan NC, De Santis M, Bruins HM, Hernandez V, Espinos EL, Dunn J, Rouanne M, et al. Updated 2016 EAU guidelines on muscle-invasive and metastatic bladder Cancer. Eur Urol. 2017;71:462-75.

6. Babjuk M. Trends in bladder Cancer incidence and mortality: success or disappointment? Eur Urol. 2017;71:109-10. 
7. Abufaraj M, Dalbagni G, Daneshmand S, Horenblas S, Kamat AM, Kanzaki R, Zlotta AR, Shariat SF. The role of surgery in metastatic bladder Cancer: a systematic review. Eur Urol. 2018;73:543-57.

8. Babjuk M. Bladder Cancer in the elderly. Eur Urol. 2018;73:51-52.

9. Shi X, Sun M, Liu H, Yao Y, Song Y. Long non-coding RNAs: a new frontier in the study of human diseases. Cancer Lett. 2013;339:159-66.

10. Trimarchi T, Bilal E, Ntziachristos P, Fabbri G, Dalla-Favera R, Tsirigos A, Aifantis I. Genome-wide mapping and characterization of notch-regulated long noncoding RNAs in acute leukemia. Cell. 2014;158:593-606.

11. Pefanis E, Wang J, Rothschild G, Lim J, Kazadi D, Sun J, Federation A, Chao J, Elliott O, Liu ZP, et al. RNA exosome-regulated long non-coding RNA transcription controls super-enhancer activity. Cell. 2015;161:774-89.

12. Cheng W, Zhang Z, Wang J. Long noncoding RNAs: new players in prostate cancer. Cancer Lett. 2013;339:8-14.

13. Fang XY, Pan HF, Leng RX, Ye DQ. Long noncoding RNAs: novel insights into gastric cancer. Cancer Lett. 2015;356:357-66.

14. Tang Y, Cheung BB, Atmadibrata B, Marshall GM, Dinger ME, Liu PY, Liu T. The regulatory role of long noncoding RNAs in cancer. Cancer Lett. 2017;391:12-9.

15. Ma Y, Zhang J, Wen L, Lin A. Membrane-lipid associated IncRNA: a new regulator in cancer signaling. Cancer Lett. 2018;419:27-9.

16. Liu D, Li Y, Luo G, Xiao X, Tao D, Wu X, Wang M, Huang C, Wang L, Zeng F, Jiang G. LncRNA SPRY4-IT1 sponges miR-101-3p to promote proliferation and metastasis of bladder cancer cells through up-regulating EZH2. Cancer Lett. 2017;388:281-91.

17. Zhan Y, Lin J, Liu Y, Chen M, Chen X, Zhuang C, Liu L, Xu W, Chen Z, He A, et al. Up-regulation of long non-coding RNA PANDAR is associated with poor prognosis and promotes tumorigenesis in bladder cancer. J Exp Clin Cancer Res. 2016;35:83.

18. Xie H, Liao X, Chen Z, Fang Y, He A, Zhong Y, Gao Q, Xiao H, Li J, Huang W, Liu Y. LnCRNA MALAT1 inhibits apoptosis and promotes invasion by antagonizing miR-125b in bladder Cancer cells. J Cancer. 2017:8:3803-11.

19. Pan J, Li X, Wu W, Xue M, Hou H, Zhai W, Chen W. Long non-coding RNA UCA1 promotes cisplatin/gemcitabine resistance through CREB modulating miR-196a-5p in bladder cancer cells. Cancer Lett. 2016;382:64-76.

20. Hedegaard J, Lamy P, Nordentoft I, Algaba F, Hoyer S, Ulhoi BP, Vang S, Reinert T, Hermann GG, Mogensen K, et al. Comprehensive transcriptional analysis of early-stage Urothelial carcinoma. Cancer Cell. 2016;30:27-42.

21. Chen J, Miao Z, Xue B, Shan Y, Weng G, Shen B. Long non-coding RNAs in urologic malignancies: functional roles and clinical translation. J Cancer. 2016:7:1842-55.

22. Andrew AS, Marsit CJ, Schned AR, Seigne JD, Kelsey KT, Moore JH, Perreard L, Karagas MR, Sempere LF. Expression of tumor suppressive microRNA-34a is associated with a reduced risk of bladder cancer recurrence. Int J Cancer. 2015;137:1158-66.

23. Liu Y, Zhang M, Liang L, Li J, Chen YX. Over-expression of IncRNA DANCR is associated with advanced tumor progression and poor prognosis in patients with colorectal cancer. Int J Clin Exp Pathol. 2015;8:11480-4.

24. Tong X, Gu PC, Xu SZ, Lin XJ. Long non-coding RNA-DANCR in human circulating monocytes: a potential biomarker associated with postmenopausal osteoporosis. Biosci Biotechnol Biochem. 2015;79:732-7.

25. Chen L, Song Z, Huang S, Wang R, Qin W, Guo J, Lin Z. IncRNA DANCR suppresses odontoblast-like differentiation of human dental pulp cells by inhibiting wnt/beta-catenin pathway. Cell Tissue Res. 2016;364:309-18.

26. Lennox KA, Behlke MA. Cellular localization of long non-coding RNAs affects silencing by RNAi more than by antisense oligonucleotides. Nucleic Acids Res. 2016;44:863-77.

27. Karlsson O, Rodosthenous RS, Jara C, Brennan KJ, Wright RO, Baccarelli AA Wright RJ: Detection of long non-coding RNAs in human breastmilk extracellular vesicles: implications for early child development. Epigenetics 2016:0. [Epub ahead of print].

28. Ma X, Wang X, Yang C, Wang Z, Han B, Wu L, Zhuang L. DANCR acts as a diagnostic biomarker and promotes tumor growth and metastasis in hepatocellular carcinoma. Anticancer Res. 2016;36:6389-98.

29. Yuan SX, Wang J, Yang F, Tao QF, Zhang J, Wang LL, Yang Y, Liu H, Wang $\mathrm{ZG}, \mathrm{Xu} \mathrm{QG}$, et al. Long noncoding RNA DANCR increases stemness features of hepatocellular carcinoma by derepression of CTNNB1. Hepatology. 2016;63:499-511.

30. Jiang N, Wang X, Xie X, Liao Y, Liu N, Liu J, Miao N, Shen J, Peng T. IncRNA DANCR promotes tumor progression and cancer stemness features in osteosarcoma by upregulating AXL via miR-33a-5p inhibition. Cancer Lett. 2017:405:46-55.
31. Mao Z, Li H, Du B, Cui K, Xing Y, Zhao X, Zai S. LncRNA DANCR promotes migration and invasion through suppression of IncRNA-LET in gastric cancer cells. Biosci Rep. 2017;37(6).

32. Sha S, Yuan D, Liu Y, Han B, Zhong N. Targeting long non-coding RNA DANCR inhibits triple negative breast cancer progression. Biol Open. 2017:6:1310-6.

33. Zhang L, Sun X, Chen S, Yang C, Shi B, Zhou L, Zhao J. Long noncoding RNA DANCR regulates miR-1305-Smad 4 axis to promote chondrogenic differentiation of human synovium-derived mesenchymal stem cells. Biosci Rep. 2017;37(4).

34. Zhang L, Yang C, Chen S, Wang G, Shi B, Tao X, Zhou L, Zhao J. Long noncoding RNA DANCR is a positive regulator of proliferation and Chondrogenic differentiation in human Synovium-derived stem cells. DNA Cell Biol. 2017;36:136-42.

35. Fan X, Yuan J, Xie J, Pan Z, Yao X, Sun X, Zhang P, Zhang L. Long nonprotein coding RNA DANCR functions as a competing endogenous RNA to regulate osteoarthritis progression via miR-577/SphK2 axis. Biochem Biophys Res Commun. 2018;500:658-64.

36. Jeronimo C, Henrique R. Epigenetic biomarkers in urological tumors: a systematic review. Cancer Lett. 2014;342:264-74.

37. Robertson AG, Kim J, Al-Ahmadie H, Bellmunt J, Guo G, Cherniack AD, Hinoue T, Laird PW, Hoadley KA, Akbani R, et al. Comprehensive Molecular Characterization of Muscle-Invasive Bladder Cancer. Cell. 2017;171:540-56 e525.

38. Maia MC, Grivas P, Agarwal N, Pal SK. Circulating tumor DNA in bladder Cancer: novel applications and future directions. Eur Urol. 2018;73:541-42.

39. Antoni S, Ferlay J, Soerjomataram I, Znaor A, Jemal A, Bray F. Bladder Cancer incidence and mortality: a global overview and recent trends. Eur Urol. 2017:71:96-108.

40. Roupret M. Words of wisdom. Re: Finasteride Reduces the Risk of Bladder Cancer in a Large Prospective Screening Study. Eur Urol. 2016;69:537.

41. Harris H. History: non-coding RNA foreseen 48 years ago. Nature. 2013:497:188

42. Joung J, Engreitz JM, Konermann S, Abudayyeh OO, Verdine VK, Aguet F, Gootenberg JS, Sanjana NE, Wright JB, Fulco CP, et al. Genome-scale activation screen identifies a IncRNA locus regulating a gene neighbourhood. Nature. 2017:548:343-6.

43. Meng L, Ward AJ, Chun S, Bennett CF, Beaudet AL, Rigo F. Towards a therapy for Angelman syndrome by targeting a long non-coding RNA. Nature. 2015;518:409-12.

44. Hon CC, Ramilowski JA, Harshbarger J, Bertin N, Rackham OJ, Gough J, Denisenko E, Schmeier S, Poulsen TM, Severin J, et al. An atlas of human long non-coding RNAs with accurate 5' ends. Nature. 2017;543:199-204.

45. Yildirim E, Kirby JE, Brown DE, Mercier FE, Sadreyev Rl, Scadden DT, Lee JT. Xist RNA is a potent suppressor of hematologic cancer in mice. Cell. 2013;152:727-42

46. Fu Z, Chen C, Zhou Q, Wang Y, Zhao Y, Zhao X, Li W, Zheng S, Ye H, Wang $L$, et al. LncRNA HOTTIP modulates cancer stem cell properties in human pancreatic cancer by regulating HOXA9. Cancer Lett. 2017:410:68-81.

47. Li C, Wan L, Liu Z, Xu G, Wang S, Su Z, Zhang Y, Zhang C, Liu X, Lei Z, Zhang HT. Long non-coding RNA XIST promotes TGF-beta-induced epithelial-mesenchymal transition by regulating miR-367/141-ZEB2 axis in non-small-cell lung cancer. Cancer Lett. 2018;418:185-95.

48. Wang Y, Liu Z, Yao B, Li Q, Wang L, Wang C, Dou C, Xu M, Liu Q, Tu K. Long non-coding RNA CASC2 suppresses epithelial-mesenchymal transition of hepatocellular carcinoma cells through CASC2/miR-367/FBXW7 axis. Mol Cancer. 2017;16:123.

49. Li J, Zhou L. Overexpression of InCRNA DANCR positively affects progression of glioma via activating Wnt/beta-catenin signaling. Biomed Pharmacother. 2018:102:602-7.

50. Lu QC, Rui ZH, Guo ZL, Xie W, Shan S, Ren T. LncRNA-DANCR contributes to lung adenocarcinoma progression by sponging miR-496 to modulate mTOR expression. J Cell Mol Med. 2018:22:1527-37.

51. Lu Y, Hu Z, Mangala LS, Stine ZE, Hu X, Jiang D, Xiang Y, Zhang Y, Pradeep $\mathrm{S}$, Rodriquez-Aguayo $\mathrm{C}$, et al. MYC targeted long noncoding RNA DANCR promotes Cancer in part by reducing p21 levels. Cancer Res. 2018;78:64-74.

52. Ma Y, Zhou G, Li M, Hu D, Zhang L, Liu P, Lin K. Long noncoding RNA DANCR mediates cisplatin resistance in glioma cells via activating AXL/PI3K Akt/NF-kappaB signaling pathway. Neurochem Int. 2018;118:233-41.

53. Tang Z, Gong Z, Sun X. LncRNA DANCR involved osteolysis after total hip arthroplasty by regulating FOXO1 expression to inhibit osteoblast differentiation. J Biomed Sci. 2018;25:4. 
54. Thin KZ, Liu X, Feng X, Raveendran S, Tu JC. LncRNA-DANCR: a valuable cancer related long non-coding RNA for human cancers. Pathol Res Pract. 2018;214:801-5.

55. Zhen Q, Gao LN, Wang RF, Chu WW, Zhang YX, Zhao XJ, Lv BL, Liu JB. LncRNA DANCR promotes lung Cancer by sequestering miR-216a. Cancer Control. 2018;25:1073274818769849.

56. Wang Y, Zeng X, Wang N, Zhao W, Zhang X, Teng S, Zhang Y, Lu Z. Long noncoding RNA DANCR, working as a competitive endogenous RNA, promotes ROCK1-mediated proliferation and metastasis via decoying of miR-335-5p and miR-1972 in osteosarcoma. Mol Cancer. 2018;17:89.

57. Kudinov AE, Karanicolas J, Golemis EA, Boumber Y. Musashi RNA-binding proteins as Cancer drivers and novel therapeutic targets. Clin Cancer Res. 2017:23:2143-53.

58. Kang MH, Jeong KJ, Kim WY, Lee HJ, Gong G, Suh N, Gyorffy B, Kim S, Jeong SY, Mills GB, Park YY. Musashi RNA-binding protein 2 regulates estrogen receptor 1 function in breast cancer. Oncogene. 2017;36:1745-52.

Ready to submit your research? Choose BMC and benefit from:

- fast, convenient online submission

- thorough peer review by experienced researchers in your field

- rapid publication on acceptance

- support for research data, including large and complex data types

- gold Open Access which fosters wider collaboration and increased citations

- maximum visibility for your research: over $100 \mathrm{M}$ website views per year

At $\mathrm{BMC}$, research is always in progress.

Learn more biomedcentral.com/submissions 